\title{
SYNCHRONIZATION OF PERIODIC SELF-OSCILLATIONS BY COMMON NOISE
}

\author{
D. S. Goldobin ${ }^{1,2 *}$ and A.S. Pikovsky ${ }^{2}$
}

UDC 537.86:519.2

We consider the effect of external noise on the stability properties of self-oscillations. A stochastic equation for the phase is derived at the limit of weak noise (in the appropriate sense). The stationary probability-density distribution is used for an analytic calculation of the Lyapunov exponent. We show that the exponent is always negative for the small noise level, which corresponds to synchronization of self-oscillations.

\section{INTRODUCTION}

The main effect of noise acting on periodic self-oscillations is the appearance of diffusion of the phase, which means that the self-oscillations cease to be ideal [1]. However, noise can also play an ordering role, in particular, synchronize self-oscillations. If two identical (or slightly different) systems are subject to common noise, then their states can be synchronized under the action of this noise. This effect is determined by the sign of the maximum Lyapunov exponent. In the case of periodic self-oscillations, it corresponds to the direction along the limit cycle. This exponent is zero for autonomous systems, so synchronization in the above-mentioned sense is absent. The Lyapunov exponent can become negative under the action of noise, which means synchronization. A similar problem was expounded for the first time in [2] in which a weakly nonlinear quasi-harmonic self-oscillator with noise in the form of a random pulse sequence was considered. The present paper deals with more general dynamical systems with white Gaussian noise.

Our approach is based on reducing the dynamics of a self-oscillating system to an equation for the phase. This is expedient if the external forcing is small in the appropriate sense. We derive a stochastic equation for the phase and find the stationary phase distribution. The Lyapunov exponent is expressed in terms of an integral over this distribution. In particular, we show that if the noise intensity is small, then this exponent is always negative, which corresponds to synchronization.

\section{BASIC MODEL}

We start from general stochastic equations

$$
\frac{\mathrm{d} x_{j}}{\mathrm{~d} t}=f_{j}(\mathbf{x})+\sum_{k=1}^{M} Q_{j k}(\mathbf{x}) \xi_{k}(t)
$$

describing the dynamics of an $N$-dimensional oscillatory system $x_{j}$, where $j=1, \ldots, N$, in the presence of mutually uncorrelated vector noise forcings $\xi_{k}(t)$ with the amplitudes $Q_{j k}$, where $k=1, \ldots, M \leq N$.

If a cycle $\mathbf{x}^{0}=\mathbf{x}^{0}\left(t+2 \pi / \omega_{0}\right)$ exists for the dynamical system without noise, then the states on this cycle can be parameterized by the phase $\varphi\left(\mathbf{x}^{0}\right)[3]$ which increases linearly with time: $\mathrm{d} \varphi / \mathrm{d} t=\omega_{0}$. The

\footnotetext{
* goldds@mail.ru
}

${ }^{1}$ Perm State University, Perm, Rusia; ${ }^{2}$ University of Potsdam, Potsdam, Germany. Translated from Izvestiya Vysshikh Uchebnykh Zavedenii, Radiofizika, Vol. 47, Nos. 10-11, pp. 1013-1019, October-November, 2004. Original article submitted May 18, 2004. 
phase $\varphi$ for a limit cycle can also be introduced in a finite vicinity of the cycle, where the relationship $\mathrm{d} \varphi / \mathrm{d} t=\omega_{0}$ also holds, without using nonuniform time renormalization undesirable for non-delta-correlated noise. With allowance for noise, evolution of the phase in a small vicinity of the cycle is described by the equation

$$
\frac{\mathrm{d} \varphi}{\mathrm{d} t}=\omega_{0}+\left.\sum_{j=1}^{N} \sum_{k=1}^{M} \frac{\partial \varphi(\mathbf{x})}{\partial x_{j}} Q_{j k}(\mathbf{x})\right|_{\mathbf{x}=\mathbf{x}^{0}(\varphi)} \xi_{k}(t) .
$$

The evolution of the system will take place in a small vicinity of the cycle in two cases: either for small intensity of noise or for finite intensity of noise and large modulus of the negative leading Lyapunov exponent. The cycle frequency can be made equal to unity by applying uniform normalization $[t]=\omega_{0}^{-1}$ of the time, which stipulates renormalization of the quantitative parameters of noise, but does not change its character. The form of the stochastic equation for the phase strongly depends on the form of noise forcing in system (1). If the single independent noise signal exists in the system, i.e., the noise forcing $\xi_{k} \neq 0$ for only one value of $k$, then we obtain

$$
\frac{\mathrm{d} \varphi}{\mathrm{d} t}=1+\varepsilon f(\varphi) \xi(t)
$$

where $\xi(t)$ will be assumed $\delta$-correlated Gaussian noise with zero mean $\langle\xi(t)\rangle=0$, which is normalized such that $\left\langle\xi(t) \xi\left(t^{\prime}+t\right)\right\rangle=2 \delta\left(t^{\prime}\right)$, the parameter $\varepsilon$ describes the noise intensity (note that $\varepsilon \sim \omega_{0}^{-1 / 2}$ upon normalization of the time), and $f(\varphi)$ is a normalized periodic function of the phase: $f(\varphi)=f(\varphi+2 \pi)$

and $\int_{0}^{2 \pi} f^{2}(\varphi) \mathrm{d} \varphi=2 \pi$. A more complicated equation is obtained if the noise signal in the initial system comprises a few independent components (this will be called the case of multicomponent noise):

$$
\frac{\mathrm{d} \varphi}{\mathrm{d} t}=1+\sum_{k=1}^{M} \varepsilon_{k} f_{k}(\varphi) \xi_{k}(t), \quad\left\langle\xi_{i}(t) \xi_{k}\left(t^{\prime}+t\right)\right\rangle=2 \delta\left(t^{\prime}\right) \delta_{i k}
$$

Here, $\delta_{i k}$ is the Kronecker delta.

Our study is aimed at analytical analysis of stability of the solutions of stochastic equations (3) and (4). For this purpose we consider Eq. (3) linearized for a small phase deviation $\alpha$ :

$$
\frac{\mathrm{d} \alpha}{\mathrm{d} t}=\varepsilon \alpha f^{\prime}(\varphi) \xi(t)
$$

Here, the prime denotes a derivative with respect to the argument. The mean rate of exponential increase in the deviation $\alpha$ is specified by the Lyapunov exponent determined by averaging the corresponding instantaneous rate:

$$
\lambda=\left\langle\frac{\mathrm{d} \ln \alpha}{\mathrm{d} t}\right\rangle=\left\langle\varepsilon f^{\prime}(\varphi) \xi(t)\right\rangle .
$$

In the case of multicomponent noise, the formula for the Lyapunov exponent has the form

$$
\lambda=\sum_{k=1}^{M}\left\langle\varepsilon_{k} f_{k}^{\prime}(\varphi) \xi_{k}(t)\right\rangle .
$$

Note that the Lyapunov exponent determines asymptotic behavior of small perturbations of the solution. Concerning our problem, it specifies whether close states of the system converge or diverge in the course of its temporal evolution. Of course, close states can diverge at some time instants even if the Lyapunov exponent is negative, but this does not prevent the overall convergence of these states. If the Lyapunov exponent is positive, then, vice versa, the states can converge in certain periods of time.

\section{FOKKER-PLANCK EQUATION AND ITS STATIONARY SOLUTION}

The Fokker-Planck equation for stochastic equation (3) in the Stratonovich sense is written in the standard way $[4,5]$ : 


$$
\frac{\partial W(\varphi, t)}{\partial t}+\frac{\partial}{\partial \varphi}\left[W(\varphi, t)-\varepsilon^{2} f(\varphi) \frac{\partial}{\partial \varphi}[f(\varphi) W(\varphi, t)]\right]=0 .
$$

The probability flow $S$ is constant in the stationary regime:

$$
W(\varphi)-\varepsilon^{2} f(\varphi) \frac{\mathrm{d}}{\mathrm{d} \varphi}[f(\varphi) W(\varphi)]=S .
$$

This makes it possible to find the solution in quadratures which has the following form for periodic boundary conditions for $W(\varphi)$ :

$$
W(\varphi)=C \int_{\varphi}^{\varphi+2 \pi} \frac{\mathrm{d} \psi}{f(\varphi) f(\psi)} \exp \left(-\frac{1}{\varepsilon^{2}} \int_{\varphi}^{\psi} \frac{\mathrm{d} \theta}{f^{2}(\theta)}\right) .
$$

Here, $C$ is found from the normalization condition for the distribution:

$$
C^{-1}=\int_{0}^{2 \pi} \mathrm{d} \varphi \int_{\varphi}^{\varphi+2 \pi} \frac{\mathrm{d} \psi}{f(\varphi) f(\psi)} \exp \left(-\frac{1}{\varepsilon^{2}} \int_{\varphi}^{\psi} \frac{\mathrm{d} \theta}{f^{2}(\theta)}\right)
$$

while the probability flow is given by the formula

$$
S=\left[1-\exp \left(-\frac{1}{\varepsilon^{2}} \int_{0}^{2 \pi} \frac{\mathrm{d} \theta}{f^{2}(\theta)}\right)\right] C
$$

Similarly, for multicomponent noise we have

$$
\frac{\partial W(\varphi, t)}{\partial t}+\frac{\partial}{\partial \varphi}\left(W(\varphi, t)-\sum_{k=1}^{M} \varepsilon_{k}^{2} f_{k}(\varphi) \frac{\partial}{\partial \varphi}\left[f_{k}(\varphi) W(\varphi, t)\right]\right)=0 .
$$

It is interesting that this equation is equivalent to its one-component analog (8) if

$$
f^{2}(\varphi)=\sum_{k=1}^{M} \varepsilon_{k}^{2} f_{k}^{2}(\varphi) / \sum_{k=1}^{M} \varepsilon_{k}^{2} \quad \text { and } \quad \varepsilon^{2}=\sum_{k=1}^{M} \varepsilon_{k}^{2}
$$

Thus, stationary solution given above is valid in this case, as well.

\section{THE LYAPUNOV EXPONENT}

Calculation of the Lyapunov exponent given by Eqs. (6) and (7) requires finding means of the form $\langle F(\varphi) \xi(t)\rangle$. Such means for stochastic equations (3) and (4) with $\delta$-correlated noise are obtained in the standard way [5]:

$$
\langle F(\varphi) \xi(t)\rangle=\varepsilon\left\langle F^{\prime}(\varphi) f(\varphi)\right\rangle
$$

Now we calculate the mean as the integral over the equilibrium density distribution of the phase and find the Lyapunov exponent

$$
\lambda=\varepsilon^{2}\left\langle f^{\prime \prime}(\varphi) f(\varphi)\right\rangle=\varepsilon^{2} C \int_{0}^{2 \pi} \mathrm{d} \varphi \int_{\varphi}^{\varphi+2 \pi} \mathrm{d} \psi \frac{f^{\prime \prime}(\varphi)}{f(\psi)} \exp \left(-\frac{1}{\varepsilon^{2}} \int_{\varphi}^{\psi} \frac{\mathrm{d} \theta}{f^{2}(\theta)}\right) .
$$

In a similar way, for multicomponent noise we obtain

$$
\lambda=\sum_{k=1}^{M} \varepsilon_{k}^{2} \int_{0}^{2 \pi} f_{k}^{\prime \prime}(\varphi) f_{k}(\varphi) W(\varphi) \mathrm{d} \varphi .
$$


Before proceeding to analysis of the obtained formulas in particular cases, we note that the Lyapunov exponent is always negative at the limit of weak noise. In the leading order in $\varepsilon$ we have

$$
\lambda_{0} \sim-\sum_{k=1}^{M} \frac{\varepsilon_{k}^{2}}{2 \pi} \int_{0}^{2 \pi}\left(f_{k}^{\prime}(\varphi)\right)^{2} \mathrm{~d} \varphi
$$

where $M$ can also be equal to 1 which corresponds to one-component noise.

\subsection{Example: linearly polarized uniform noise}

If only one component of the initial system is subject to additive noise, while the limit cycle is close to a circle, then we arrive at a one-component stochastic equation with $f(\varphi)=\sqrt{2} \sin \varphi$. Here, we also assume that the phase-flow velocity is approximately constant along the limit cycle. In this case,

$$
\int_{\varphi}^{\varphi+2 \pi} \frac{\mathrm{d} \psi}{f(\psi)} \exp \left(-\frac{1}{\varepsilon^{2}} \int_{\varphi}^{\psi} \frac{\mathrm{d} \theta}{f^{2}(\theta)}\right)=\int_{\varphi}^{\pi+\pi[\varphi / \pi]} \frac{\mathrm{d} \psi}{\sqrt{2} \sin \psi} \exp \left(\frac{\cot \psi-\cot \varphi}{2 \varepsilon^{2}}\right),
$$

where the square brackets denote the integer part of an expression. The distribution for the specified form of the function $f(\varphi)$ has a period $\pi$, and for $\varphi \in[0, \pi)$ we have

$$
\begin{gathered}
W(\varphi)=\frac{C}{2} \int_{\varphi}^{\pi} \frac{\mathrm{d} \psi}{\sin \psi \sin \varphi} \exp \left(\frac{\cot \psi-\cot \varphi}{2 \varepsilon^{2}}\right) \\
S=C=\left[\int_{-\infty}^{+\infty} \mathrm{d} y \int_{-\infty}^{y} \frac{\mathrm{d} x}{\sqrt{1+x^{2}} \sqrt{1+y^{2}}} \exp \left(\frac{x-y}{2 \varepsilon^{2}}\right)\right]^{-1}, \\
\lambda=-\frac{\varepsilon^{2} C}{2} \int_{-\infty}^{+\infty} \mathrm{d} y \int_{-\infty}^{y} \frac{\mathrm{d} x}{\left(1+x^{2}\right)^{1 / 2}\left(1+y^{2}\right)^{3 / 2}} \exp \left(\frac{x-y}{2 \varepsilon^{2}}\right) .
\end{gathered}
$$

To arrive at the last formulas, we introduced the variables $x=\cot \psi$ and $y=\cot \varphi$ (the transformation is necessary to make convergence of the integrals obvious). The further simplification of the formulas seems impossible, although these formulas are already quite convenient for constructing a numerical solution. The desired Lyapunov exponent as a function of the noise intensity is plotted in Fig. 1.

\subsection{Example: superposition of two independent linearly polarized uniform noises}

Within the framework of the same assumptions as those adopted above (i.e., the limit cycle of a dynamical system is close to a circle and the motion along this cycle is uniform), the effect of noise acting upon two components shifted in phase by $\pi / 2$ is naturally modeled by the multicomponent noise with $f_{1}(\varphi)=\sqrt{2} \sin \varphi$ and $f_{2}(\varphi)=\sqrt{2} \cos \varphi$. The mean probability density corresponds to the effective function $f(\varphi)=\sqrt{1+\Delta \cos (2 \varphi)}$ and the effective noise intensity $\varepsilon^{2}=\varepsilon_{1}^{2}+\varepsilon_{2}^{2}$, where $\Delta \equiv\left(\varepsilon_{2}^{2}-\varepsilon_{1}^{2}\right) /\left(\varepsilon_{2}^{2}+\varepsilon_{1}^{2}\right)$ and, obviously, $\Delta \in[-1,1]$. The form of the function $f(\varphi)$ allows us to conclude that the system possesses 
symmetry with respect to the replacement $(\Delta, \varphi) \leftrightarrow(-\Delta, \varphi+\pi / 2)$. In this case,

$$
\int \frac{\mathrm{d} \theta}{f^{2}(\theta)}=\frac{1}{\sqrt{1-\Delta^{2}}}\left(\pi\left[\frac{\theta}{\pi}\right]-\arctan \left(\sqrt{\frac{1+\Delta}{1-\Delta}} \cot \theta\right)\right),
$$

so that for the mean distribution $W(\varphi)$ and the probability flow $S$ we obtain

$$
\begin{gathered}
W(\varphi)=C \int_{\varphi}^{\varphi+\pi} \mathrm{d} \psi \frac{\exp \left(-\left.\frac{1}{\varepsilon^{2} \sqrt{1-\Delta^{2}}}\left(\pi\left[\frac{\theta}{\pi}\right]-\arctan \left(\sqrt{\frac{1+\Delta}{1-\Delta}} \cot \theta\right)\right)\right|_{\varphi} ^{\psi}\right)}{\sqrt{1+\Delta \cos (2 \varphi)} \sqrt{1+\Delta \cos (2 \psi)}}, \\
C=\left(2 \int_{0}^{\pi} \mathrm{d} \varphi \int_{\varphi}^{\varphi+\pi} \mathrm{d} \psi \frac{\exp \left(-\left.\frac{1}{\varepsilon^{2} \sqrt{1-\Delta^{2}}}\left(\pi\left[\frac{\theta}{\pi}\right]-\arctan \left(\sqrt{\frac{1+\Delta}{1-\Delta}} \cot \theta\right)\right)\right|_{\varphi} ^{\psi}\right)}{\sqrt{1+\Delta \cos (2 \varphi)} \sqrt{1+\Delta \cos (2 \psi)}}\right)^{-1}, \\
S=\left[1-\exp \left(-\frac{\pi}{\varepsilon^{2} \sqrt{1-\Delta^{2}}}\right)\right] C .
\end{gathered}
$$

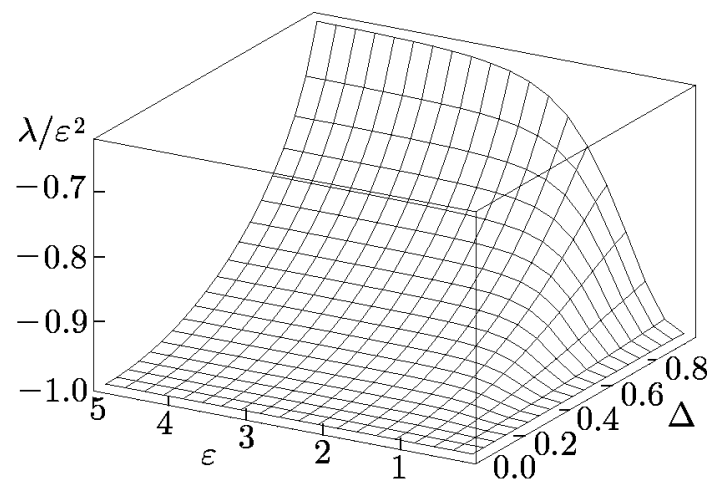

Fig. 2. Superposition of two independent linearly polarized uniform noises. The Lyapunov exponent normalized to $\varepsilon^{2}$ is plotted as a function of $\varepsilon$ and $\Delta$.
The resulting formula for the Lyapunov exponent has the form

$$
\begin{aligned}
\lambda=-2 \varepsilon^{2} C & \int_{0}^{\pi} \mathrm{d} \varphi \int_{\varphi}^{\varphi+\pi} \mathrm{d} \psi \frac{\sqrt{1+\Delta \cos (2 \varphi)}}{\sqrt{1+\Delta \cos (2 \psi)}} \\
& \times \exp \left(-\frac{1}{\varepsilon^{2} \sqrt{1-\Delta^{2}}}\left(\pi\left[\frac{\theta}{\pi}\right]\right.\right. \\
& \left.\left.-\arctan \left(\sqrt{\frac{1+\Delta}{1-\Delta}} \cot \theta\right)\right)\left.\right|_{\varphi} ^{\psi}\right) .
\end{aligned}
$$

The dependence of the desired Lyapunov exponent on the noise intensity $\varepsilon$ and the essential parameter $\Delta$ is shown in Fig. 2.

\section{CONCLUSIONS}

In this paper, we analyze the possibility of synchronization of two identical dynamical systems, admitting description in terms of the phase, by virtue of common external white Gaussian noise. The Lyapunov exponent corresponding to perturbations along a phase trajectory of the dynamical system is the quantitative characteristic of the ability for synchronization. The problem of finding this exponent is solved analytically in quadratures for the cases of one (see Eqs. (10), (11), and (16)) and several (Eq. (17)) independent noise signals. The problems of the effect of one and two independent linearly polarized uniform noises on synchronization of systems having limit cycle close to a circle and approximately constant modulus of the phase-flow velocity along this cycle (see Figs. 1 and 2) are considered as examples of the systems corresponding to the first and second cases.

In both examples considered above, noise can only lead to synchronization since the Lyapunov exponent is negative. The same conclusion follows from the general asymptotic expression valid for any system 
at the limit of weak noise. Note that the assumption of Gaussian noise is redundant at this limit. Indeed, Gaussianity is used to remove the higher cumulants of the noise signal whose contributions to the values of the desired quantities are proportional to the corresponding higher powers of the noise intensity. However, the asymptotic values of the Lyapunov exponent at this limit are linearly proportional to the noise intensity and inversely proportional to the self-oscillation frequency. The question on the sign of the Lyapunov exponent for strong noise is of undoubted interest. In this case, the full system should be analyzed numerically.

D. S. Goldobin acknowledges the support by the Germany-France-Russia Trilateral Project, the "Dynasty" Foundation, and the International Center for Fundamental Physics in Moscow.

\section{REFERENCES}

1. A. N. Malakhov, Fluctuations in Self-Oscillating Systems [in Russian], Nauka, Moscow (1968).

2. A. S. Pikovsky, Izv. Vyssh. Uchebn. Zaved., Radiofiz., 27, 390 (1984).

3. Y. Kuramoto, Chemical Oscillations, Waves and Turbulence, Springer (1984).

4. S. M. Rytov, Introduction to Statistical Radiophysics [in Russian], Nauka, Moscow (1966).

5. V.I. Klyatskin, Statistical Description of Dynamic Systems with Fluctuating Parameters [in Russian], Nauka, Moscow (1975). 